\title{
Die Historie: Anfänge und Entwicklungen der Dermatologie in Mannheim
}

\author{
The History: Beginning and Development of Dermatology in Mannheim
}

\section{Zusammenfassung}

Die Dermatologie wurde in Mannheim im Jahre 1901 eingerichtet und ist jetzt 100 Jahre alt. Sie ist gekennzeichnet durch Konstanz und Kompetenz, repräsentiert durch die 4 Chefärzte, welche großen Zeitabschnitten eine persönliche Prägung gaben und ihre Fachkompetenz zur Verfügung stellten. Diese Zeit ist aber auch gekennzeichnet durch Innovation und Flexibilität, welche in der ersten Hälfte der 100 Jahre durch die Venerologie und deren therapeutische Bewältigung gefordert wurden. Die zweite Hälfte ist geprägt durch einen Gestaltwandel, welcher die Zuwendung zur Dermatologie der Berufswelt und derjenigen der Freizeit umfasst (Umweltdermatologie) und der zunehmenden Bedeutung der Dermato-Onkologie Rechnung trägt. Getragen und gestaltet wurden diese Entwicklungen von vier leitenden Ärzten, den zwei städtischen Chefärzten Heinrich Loeb von 1901 bis 1932 und Friedrich Schmidt-LaBaume von 1932 bis 1958 sowie den beiden Ordinarien Werner Schmidt von 1958 bis 1975 und Ernst G. Jung von 1975 bis 2000. Im April 2000 hat Sergij Goerdt den Lehrstuhl, verbunden mit der Direktion der nun als „Universitätsklinik für Dermatologie, Venerologie und Allergologie“ bezeichneten Hautklinik übernommen. Ihm fällt die Aufgabe und die Chance eines „hauteigenen“ Neubaus zu, der 2005 zu beziehen geplant ist.

\section{Abstract}

Dermatology was established in Mannheim 1901 and completes now its first 100 years. It is characterized by the constancy and competence of the 4 directors, each of them representing a long period of personal skill. These 100 years are marked by innovation and flexibility, as well. The first half is dominated by venerology and its therapeutical implications. The second half represents a period of fundamental changes in the direction of environmental dermatology (in occupation as well as in leisure), also respecting the increasing relevance of dermato-oncology. Such a development of dermatology was formed and carried out by four directors: Heinrich Loeb (1901-1932), Friedrich SchmidtLaBaume (1932-1958), Werner Schmidt (1958-1975) and Ernst G. Jung (1975-2000). In April 2000 Sergij Goerdt took over the chair of dermatology as the director of the „Universitätsklinik für Dermatologie, Venerologie und Allergologie“. It is his unique and solemn chance to plan and to realize the new building, especially for dermatology, to be taken into operation during 2005.
Die Dermatologie in Mannheim hat sich 1901 mit der Einrichtung einer Hautabteilung am Allgemeinen Krankenhaus etabliert und ist jetzt 100 Jahre alt. Vier leitende Ärzte, zwei als städtische Chefärzte, zwei als Ordinarien, haben in besonderer Kontinuität diese 100 Jahre bestritten und auch geprägt. Als Fünfter in der Reihe hat Prof. Dr. Sergij Goerdt, aus Berlin nach Mannheim be- rufen, am 16. 4. 2000 die Direktion der nun als „Universitätsklinik für Dermatologie, Venerologie und Allergologie“ bezeichnete Einrichtung übernommen und führt sie tatkräftig weiter voran. Ein guter Grund, der Historie von gerade 100 Jahren im Folgenden zu gedenken.Die Voraussetzung 
Im Jahre 1701, also vor 300 Jahren, wurde von der Stadtverwaltung in Mannheim ein Nothaus in Betrieb genommen zur Beherbergung und Verpflegung von Fremden und armen Kranken. In den anschließenden Jahrzehnten entstanden Hospitäler verschiedener Glaubensbrüderschaften und das kurfürstliche KarlBorromäus-Hospital im Quadrat R 5. Die verschiedenen Standorte wurden 1807 zum „Städtischen Krankenhaus“ zusammengefasst. Weitere Entwicklungen erfolgten in R 5 durch Zukauf und Aufstockung. Im Jahre 1860 ist das Mannheimer „Allgemeine Krankenhaus" wohl das größte badische Krankenhaus [1] mit 336 Betten in 50 Räumen, einer Verweildauer von 27,3 Tagen und einer Gliederung in Stationen für innere Krankheiten, Chirurgie, Wöchnerinnen und Kinder sowie für „Krätzige und Syphilitische“. Der Anteil der Krätzekranken (Skabies) war sehr hoch (1854: 491 Fälle unter 2950 Aufnahmen und im Jahre 1858 immer noch 125 Fälle unter 2304 Aufnahmen), weshalb jeder badische Amtsbezirk besondere Krätzezimmer vorzuhalten hatte [2]. Die starke Verbreitung der Krätze wurde zurückgeführt auf die Revolutionskämpfe von 1849, die anschließende Besetzung Badens durch preußische Truppen und die Beteiligung badischer Truppenteile am deutsch-dänischen Krieg 1864 und im deutschösterreichischen Krieg 1866. Die Krätze war und blieb ein besonderes Problem, mit deutlichen Schwerpunkten in den Zeiten von Kriegen, Besetzungen und Garnisonsbelastung. Zudem lässt sich eine rhythmische, wohl immunologisch zu deutende, Skabieshäufung mit Wiederkehr alle 15-18 Jahre erkennen, die kräftig überlagert wird durch die kriegsbedingten Epidemien und, in den letzten 50 Jahren, durch kleinere und größere Epidemien, die korreliert scheinen mit den besonderen sozialen Umständen und Pflegebedingungen $[3,4]$.

Die Krätzebehandlung war 1860 als äußere, lokale Therapie klar geregelt [1]: „Das Verfahren ist das der Schnellkur, mit Schmierseife und darauf folgender Schwefelkalisalbe. Die Krätzigen werden in der Anstalt nicht verpflegt, sondern nur behandelt und nach Verlauf von einigen Stunden geheilt entlassen. Die Anstalt bedarf daher keiner Verköstigung und keiner Betten." Die Taxe für diese Kur war nach Vermöglichkeit gestaffelt und für Ausländer besonders hoch.

Klar ist erkennbar, dass damals schon soziale Überlegungen die Finanzierung strukturierten, dass die Vorteile einer „Tagesklinik“ erkannt und genützt wurden und dass zwischen dem medizinischen Alltag und den offiziellen Berichten enorme Diskrepanzen bestehen. Auch die modernen Möglichkeiten der Skabiesbehandlung, neuerdings sogar systemisch, vermögen den vor 140 Jahren formulierten Traum der tagesambulanten, zuverlässigen Heilbehandlung in wenigen Stunden nicht zu realisieren.

Die Raumverhältnisse im Allgemeinen Krankenhaus in $\mathrm{R} 5$ wirkten immer stärker beengend, so dass an ein neues, modernes Krankenhaus an anderer Stelle gedacht wurde. Ein erster Antrag erfolgte bereits 1889. Jahre verstrichen, in welchen der Bedarf, die Standortfrage, die Finanzierung, die Gliederung und die Organisation wiederholt zur Debatte standen. Erst am 13.12.1912 genehmigte die Mannheimer Bürgerschaft die Neubaupläne am Neckarufer. Im Jahre 1913 erfolgte die Grundsteinlegung, während der Neubau, verzögert durch den Weltkrieg 1914-18, erst 1922 fertiggestellt und in Betrieb genommen werden konnte. Deutlich früher wurden zwei neue Fachabteilungen errichtet: die Augenabteilung 1889 und die Hautabteilung 1901, die vorerst noch im Allgemeinen Krankenhaus in R 5 (insgesamt 540 Betten mit einer Station für Geschlechtskranke) untergebracht wurden (in Heidelberg wurde 1908, in Ludwigshafen 1910 eine Hautklinik etabliert). Im Neubau am Neckarufer mit damals 1040 Krankenbetten wurden für die Hautabteilung zwei Pavillons im Park eingerichtet, das jetzige Haus 24 mit seinerzeit 98 Betten für Hautkranke und das Haus 26 mit 82 Betten für geschlechtskranke Prostituierte. Der Umzug erfolgte mit einem Krankenstand von 32 Hautpatienten und 40 Prostituierten [5].

Die Ausgangslage zur Gründung einer Abteilung für Hautkranke, die am 28. 3.1901 vom Stadtrat auf Antrag des Krankenhausausschusses genehmigt wurde, waren also zum einen die wiederkehrenden Krätze-Epidemien und zum anderen das therapeutisch und epidemiologisch ungelöste Problem der Geschlechtskrankheiten.

\section{Der Anfang durch Venerologie und Hautinfekte}

Die Abteilung für Hautkranke wurde nach der Genehmigung durch den Stadtrat am 28. 3.1901 noch im selben Jahr am Allgemeinen Krankenhaus in $\mathrm{R} 5$ eingerichtet. Die Leitung erhielt Herr Dr. med. Heinrich Loeb (geb. am 1.1.1865 in Edenkoben in der Pfalz, gest. am 1.5.1932 in Mannheim).

Die Studienjahre verbrachte er an der Universität Straßburg, wo

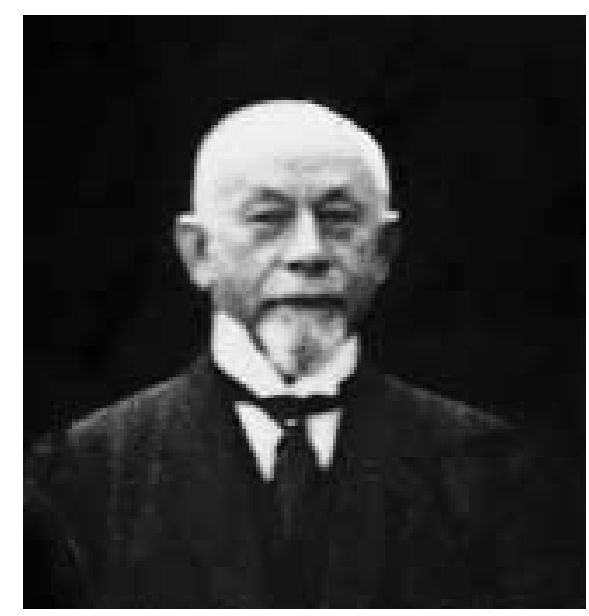

Abb. 1 Dr. med. Heinrich Loeb (1865 - 1932) anlässlich der Jahrestagung der Vereinigung Südwestdeutscher Dermatologen im März 1932 in Mannheim.

er nach dem medizinischen Staatsexamen, nach anfänglichem Zögern, sich dem Spezialfach der Dermatologie zuwandte und als Assistent von Prof. Alfred Wolff (1850-1916) Facharzt für Haut- und Geschlechtskrankheiten wurde. 1892 ließ er sich als solcher in Mannheim M 7,17 nieder. Im Jahre 1893 erhielt er die Erlaubnis zur Abhaltung eines Ambulatoriums für unbemittelte Hautkranke im Allgemeinen Krankenhaus in R 5. 1901 wurde die Hautabteilung eingerichtet und Dr. Loeb als leitender Arzt bestellt, wobei er zunächst seine Hautarztpraxis in der Stadt weiterführte (Abb.1). Aus primitiven Anfängen schuf er schrittweise eine moderne dermatologische Abteilung, die diagnostisch und therapeutisch alles bot, was damals möglich war. Erfinderisch schuf er neben der „Loebschen Spritze“ noch eine ganze Reihe von Neuheiten technischer Art. Er betrieb die Dermatologie im Verbund mit den anderen medizinischen Fächern, die Haut 
auch als Spiegel innerer Krankheiten zu werten, beschäftigte sich schon mit Allergien und beschrieb als Erster die progressive Pigmentpurpura (Adalinexanthem).

1910 nahm er Martin Friedmann als Kompagnon in die Praxis und widmete sich fortan hauptamtlich seiner Hautabteilung und mit besonderem Impetus der Venerologie. Enge Beziehungen zu Paul Ehrlich ermöglichten ihm schon 1910 die frühe und effektvolle Einführung des Salvarsans in die Syphilisbehandlung. Bei der Bekämpfung der Gonorrhö (Tripper) verbesserte und ergänzte er die damals noch unbefriedigende lokale Behandlung. Seine Methode der Abortivbehandlung der männlichen und weiblichen Gonorrhö, die Präventivbehandlung der Frau, die intensive Milchfieberbehandlung und sein Sammelvakzin brachten ihn seinem Ziele näher, die Heilungsdauer zu verkürzen.

Er richtete die Beratungsstelle für Geschlechtskranke in Mannheim ein und etablierte mit dem „Mannheimer System“ die regelmäßige und effiziente Kontrolle der Prostituierten durch ihre Ärzte. Dies wurde teilweise in das Gesetz zur Bekämpfung der Geschlechtskrankheiten übernommen. Er war Gründungsmitglied der Deutschen Gesellschaft zur Bekämpfung der Geschlechtskrankheiten, Vorstandsmitglied des entsprechenden Landesverbandes Baden sowie Gründer und über drei Jahrzehnte Schriftführer der Ortsgruppe Mannheim.

Dr. Heinrich Loeb war ein fröhlicher, geistreicher, naturverbundener Mensch mit pfälzischem Mutterwitz, beliebt unter Kollegen und hochgeschätzt bei seinen Patienten. Zu seiner großen Freude, war es ihm vergönnt, am 5. und 6.3.1932 die Tagung der Vereinigung südwestdeutscher Dermatologen in Mannheim durchzuführen. Am 1.5.1932 verstarb er nach einem Pfalzausflug unerwartet und plötzlich an einem Herzanfall im 68. Altersjahr [6,7]. Am 4.5.1932 nahm eine große Trauergemeinde im Krematorium Abschied von Dr. Heinrich Loeb, Mannheims berühmtem Dermatologen, wie sich der ärztliche Direktor des Krankenhauses, Prof. Dr. Karl Kießling, ausdrückte.

Noch im selben Jahr wurde als Nachfolger Loeb's und Chefarzt der Hautklinik Mannheim, Herr Prof. Dr. med. Friedrich SchmidtLaBaume, gewählt (geb. am 19.4.1892 in Wittenberg, gest. am 5.10.1973 in Baden-Baden). Er studierte Medizin in Berlin, wo er nach Unterbrechung durch den Kriegsdienst als Feldunterarzt im Jahre 1919 das Staatsexamen ablegte. Nach 5 Jahren breiter medizinischer Ausbildung wechselte er an die UniversitätsHautklinik Frankfurt (Main). Dort erfuhr er in den Jahren 1926-1930 unter Prof. Karl Herxheimer eine breite dermato-venerologische Ausbildung. Er habilitierte sich mit einem Thema zur Dermoelastometrie 1928 in Frankfurt und war in den Jahren 1930-1932 unter dem neuen Chef Prof. Oskar Gans ebendort als Privatdozent und Oberarzt tätig. Seinen Dienst als Chefarzt der Mannheimer Hautklinik nahm er am 15.9.1932 auf. Im Jahre 1939 wurde er in Frankfurt zum apl. Professor ernannt (Abb. 2).

Einer seiner klinischen und wissenschaftlichen Schwerpunkte war die Venerologie, die in den Jahren nach dem 2. Weltkrieg in Mannheim eine überragende Bedeutung bekam. 1945 und in den nachfolgenden Jahren wurde das Städt. Krankenhaus von Patienten mit Geschlechtskrankheiten regelrecht überflutet. Die Hautklinik konnte die enorme Zahl Geschlechtskranker, vorwiegend mit Gonorrhö (Tripper), nicht mehr fassen, so dass die be-

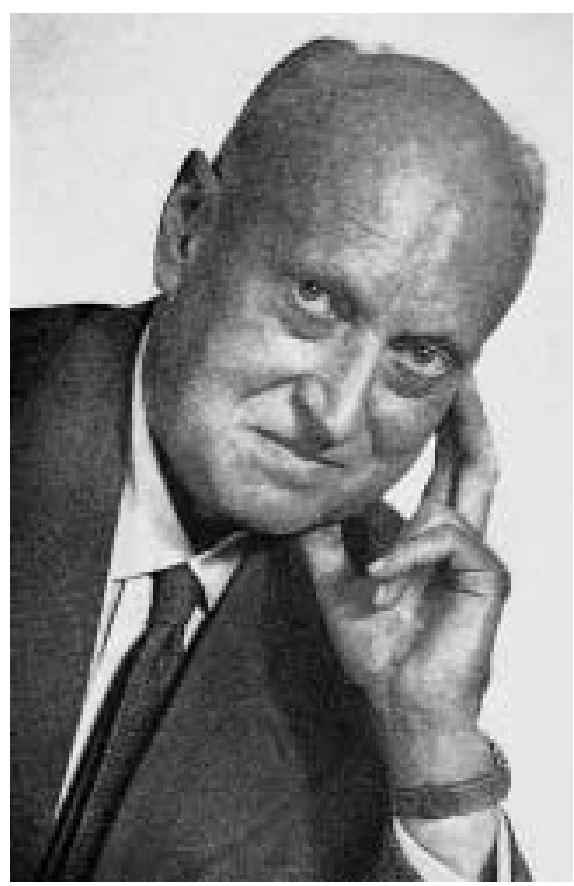

Abb. 2 Prof. Dr. med. Friedrich Schmidt-LaBaume (1892-1958).

nachbarten Kliniken Stationen abtreten mussten. Zudem wurden im Park Baracken aufgestellt, bis die Hautklinik im Jahre 1946 vorübergehend über 400 Betten verfügte. Die Rationalisierung der Gonorrhöbehandlung mit Verkürzung der Liegedauer war eines seiner großen Anliegen, welches er dank der guten Beziehungen zu den Sanitätsoffizieren der amerikanischen Garnison durch deren Penicillin erreichte. Die junge Mannheimer Firma C. F. Boehringer \& Söhne $\mathrm{GmbH}$ tat das Ihrige, indem sie aus dem Sammelurin der mit Penicillin behandelten Patienten das Medikament wieder zurückgewann und für andere Patienten bereitstellte. Die Beratungsstelle zur Kontrolle der Prostituierten verblieb noch lange in $\mathrm{R} 5$.

Er war jahrzehntelang Vorstandsmitglied der Deutschen Dermatologischen Gesellschaft und der Landesgruppe Baden der Deutschen Gesellschaft zur Bekämpfung der Geschlechtskrankheiten, er gründete die Vereinigung Mannheimer und Ludwigshafener Dermatologen und hatte die Ehre, im Mai 1954 die 75. Tagung der Vereinigung Südwestdeutscher Dermatologen in Mannheim mit über 350 Teilnehmern durchzuführen (Abb.3). Prof. Fr. Schmidt-LaBaume galt im Nachkriegsdeutschland als einer der prominentesten und meistbekannten Dermatologen, nachdem er, bis zur politischen Entlastung, als Chefarzt vom 13.12.1945-20.3.1947 vorübergehend entlassen und solange im gewöhnlichen Arbeitsverhältnis weiterbeschäftigt worden ist. Er hielt der Stadt Mannheim, die Pavillons wiesen im Gegensatz zum Haupthaus wenig Kriegsschäden auf, die Treue, obschon er wiederholt eingeladen wurde, zerstörte UniversitätsHautkliniken wiederaufzubauen $[8,9]$. In diesen Jahren wurde die Hautklinik durch die Errichtung bakteriologischer, mykologischer und histologischer Laboratorien ergänzt. Sprechstunden zur Kontrolle der Hauttuberkulose (sog. Lupus-Sprechstunden) wurden flächendeckend in der Region etabliert. Die Epikutantestung wurde eingeführt und eine allergenarme Kammer eingerichtet. 


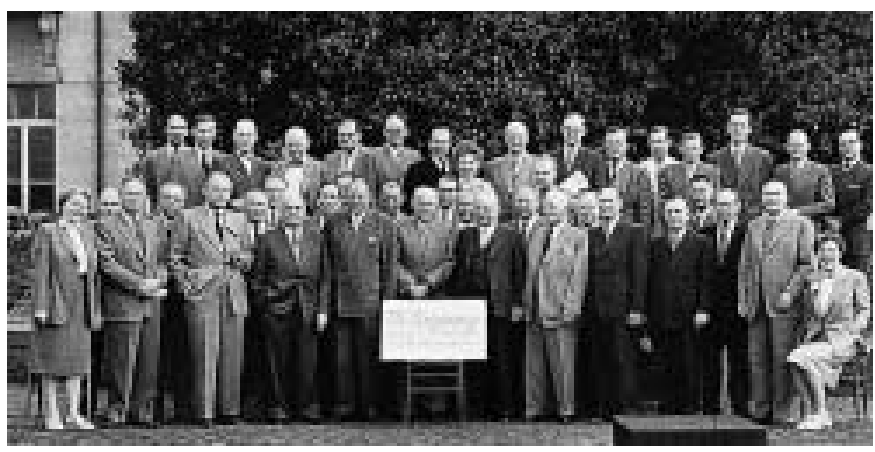

Abb. 3 Prof. Friedrich Schmidt-LaBaume anlässlich der 75. Jahrestagung der Vereinigung Südwestdeutscher Dermatologen 1954 in Mannheim, umgeben von seinen Kollegen Proff. Schönfeld, Marchionini (li.) und Gans, Gottron, Hämel und Memmesheimer.

Ein zweiter Schwerpunkt von Prof. Schmidt-LaBaume war die Berufsdermatologie, wobei er sehr früh erkannte, dass mit dem Wiederaufbau und dem Blühen der Mannheimer Schwerindustrie, dem Ausbau des Hafens und dem gewaltigen Chemieboom in der Region neuartige Belastungen der Haut, besonders an den Händen und im Gesicht, auftreten werden. Diese sind einerseits bedingt durch aggressive Arbeitsstoffe und Verfahren, anderseits aber nicht minder durch die dadurch nötige Reinigung. Hautschutz am Arbeitsplatz, adäquate Reinigung und ausreichende Hautpflege waren die Probleme der Jahre für Tausende von Arbeitern, zu differenzieren entsprechend dem Arbeitsplatz und unter Berücksichtigung der individuellen Beschaffenheit der Haut. In diese Zeit fielen auch die schweren Dioxin-Vergiftungen durch Chemie-Unfälle mit, unter anderem, früh manifesten, chronisch progredienten Hautveränderungen (sog. „DioxinAkne") und die Kleinepidemien mit erworbener Vitiligo durch Arbeitsstoffe wie melanotoxische Hydrochinone.

Den dritten Schwerpunkt setzte er mit seinen Arbeiten zur lokalen Dermatopharmakologie und -therapie, wobei er Stabilität, Wirkungsnachweis, Sterilität und Verträglichkeit als Kriterien einsetzte und so die Umstellung von der individuellen Rezeptur auf moderne Fertigpräparate einleitete. Seit 1939 hat er diesen grundlegenden Erfahrungsschatz in Büchern verankert, die mehrere Auflagen erfuhren und viele Jahrzehnte lang die Richtung wiesen $[10,11]$. Im Jahre 1957 erhielt er das Bundesverdienstkreuz 1. Ordnung.

Prof. Friedrich Schmidt-LaBaume leitete die Mannheimer Hautklinik 26 Jahre lang und trat nach Vollendung seines 66 . Lebensjahres am 31. Juli 1958 in den Ruhestand. Er verlegte seinen Wohnsitz nach Baden-Baden, wo er noch konsiliarisch und in seiner gepflegten Privatpraxis tätig war, bis er am 5.10.1973 im Alter von 81 Jahren verstarb.

\section{Die Umstellung}

Als Nachfolger wurde noch 1958 der ehemalige Ludwigshafener Kollege Prof. Dr. med. Werner Schmidt (geb. am 9. 4.1907 in Saarbrücken, gest. am 14.8.1989 in Baden-Baden) gewählt. Er legte das medizinische Staatsexamen 1931 in Tübingen ab, wo er 1932 auch promovierte. Die dermatologische Fachausbildung erfuhr er 1932-1934 an der städt. Hautklinik Essen (Prof. Alois
Memmesheimer) und 1934-1935 an der Univ.-Hautklinik Tübingen (Prof. Paul Linser). Von 1935 bis 1945 war er Oberarzt an der Hautklinik am Rudolf Virchow-Krankenhaus in Berlin (Prof. Heinrich Löhe), wo er sich 1939 mit einem allergologischen Thema habilitierte. Nach dem Krieg übernahm er 1946 die Venerologische Abteilung am Krankenhaus Wimbern (Ruhr), ließ sich in Stuttgart 1947 als Hautarzt nieder und versah kommissarisch die Leitung der Hautklinik Ludwigsburg. 1950 wurde er zum Chefarzt der städt. Hautklinik in Ludwigshafen (Rhein) gewählt. 1952 habilitierte er sich an die Universität Mainz um, wo 1956 auch die Ernennung zum apl. Professor erfolgte. 1958 wechselte er über den Rhein als Chefarzt der Hautklinik nach Mannheim. Auch Prof. Werner Schmidt (Abb. 4 ) brachte einen venerologischen Schwerpunkt mit, hatte er doch die enormen Behandlungserfolge der Antibiotika bei der Syphilis und der Gonorrhö mitgestaltet.

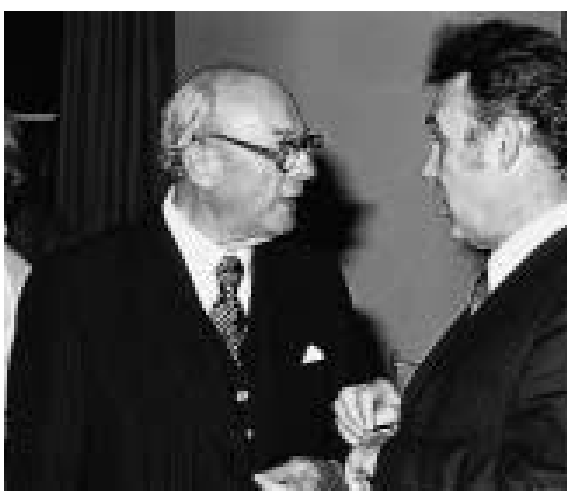

Abb. 4 Prof. Dr. med. Werner Schmidt (1907 1989) anlässlich seiner Abschiedsvorlesung am 5.11.1975, zusammen mit Bürgermeister Dr. Hans Martini, Ehrensenator der Universität Heidelberg.

Die Venerologie machte in den Jahren 1945-1960 einen enormen Wandel durch. Der Bettenbedarf für Geschlechtskranke nahm gewaltig ab, so dass der Pavillon Haus 26 an andere Kliniken abgetreten werden konnte (Psychiatrie und Kinderklinik, später Onkologie). Die ambulante Behandlung wurde vornehmlich den niedergelassenen Dermatologen übertragen und die Beratungsstelle mit der Kontrolle der Prostituierten ins staatliche Gesundheitsamt verlegt, wo sie von den dortigen Hautfachärzten übernommen wurde. So konnte sich die Hautklinik und insbesondere deren kundiger Leiter vermehrt um die komplizierten Fälle der Venerologie, um die selteneren Infektionen und in zunehmendem Maße auch um die tropischen Infektionskrankheiten kümmern, die von Gastarbeitern und auch von Mannheimern aus Fernreisen eingeschleppt wurden. Lepra, Leishmaniasis, tropische Parasiten und eine Vielzahl exotischer Mykosen bedingten eine Anpassung der Laboratorien und forderten seine speziellen Kenntnisse. Die Andrologie wurde etabliert, besonders zur Abklärung postinfektiöser Sterilität beim Manne und deren Behandlung. Folgerichtig wurde ihm der Vorsitz der Gesellschaft zur Bekämpfung der Geschlechtskrankheiten in Baden-Württemberg anvertraut.

Der zweite Schwerpunkt von Prof. Werner Schmidt setzte sich aus der Allergologie und der Berufsdermatologie zusammen. Erkenntnisse und Diagnostik allergischer Berufserkrankungen, deren Abgrenzung vom toxischen Geschehen, die Prävention durch Allergenkarenz und Hautschutz sowie die sachgerechte gutachterliche Beurteilung waren seine besonderen Anliegen, denen er auch eine große Zahl wissenschaftlicher Publikationen und Fortbildungen widmete. Der große Industrieraum Rhein-Neckar bie- 
tet sich dafür geradezu an. Prof. Werner Schmidt war einer der ersten, der klinische Therapieversuche mit oralen Antihistaminika bei Allergie erfolgreich durchführte und publizierte. Er trug den neuen Möglichkeiten der ambulanten Diagnostik und Behandlung von Hautkrankheiten Rechnung durch die Einrichtung einer effektiven Ambulanz, die konsiliarisch innerhalb des Städtischen Krankenhauses und auf Zuweisung durch niedergelassene Haut- und Spezialärzte tätig wurde. Damit war die Bettenzahl auf 78 Betten zu reduzieren.

Im November 1964 erfolgte die feierliche Eröffnung des Klinikum Mannheim durch den damaligen Ministerpräsidenten von Baden-Württemberg, Dr. Kurt Georg Kiesinger, als zweite Ausbildungsstätte für klinische Medizin an der Universität Heidelberg. Akademisch wurde in Mannheim eine Sektion der Medizinischen Fakultät Heidelberg formiert, die erst 1970 zur selbständigen „Fakultät für klinische Medizin Mannheim der Universität Heidelberg" wurde [12]. Und so ist es bis jetzt geblieben [13]. Mit einem Lehrauftrag versehen, begann Prof. Werner Schmidt die Vorlesungen und Kurse in Dermatologie und Venerologie im Jahre 1966. Im Jahre 1968 wurde er zum ordentlichen Professor unseres Faches berufen, eine Funktion, die er mit großer Begeisterung bis zu seiner Emeritierung am 30. 9.1975 ausfüllte. Er hat die Mannheimer Hautklinik zu einer vollwertigen akademischen Stätte der Lehre und Forschung entwickelt und begeisterte seine Studenten in besonderem Maße. Ein studentischer Fackelzug belohnte diesen Einsatz am Vorabend seiner akademischen Abschiedsvorlesung am 5.11.1975 [14-16].

Prof. Werner Schmidt verlegte seinen Wohnsitz bald darauf nach Baden-Baden, gleichsam dem Vorgänger nachfolgend, wo er sich der Medizingeschichte seiner Mannheimer Fakultät und der vorangehenden Krankenanstalten widmete [2-4]. Dort verstarb er nach längerer Krankheit am 14. 8.1989, aufopfernd gepflegt von seiner über alles geschätzten Gattin, die nach wie vor, auch stellvertretend für ihren Mann, am weiteren Geschick der Mannheimer Dermatologie regen Anteil nimmt [17].

\section{Neue Ziele}

Als Nachfolger wurde Prof. Dr. med. Ernst G. Jung (geb. am 3.3.1932 in Winterthur in der Schweiz) berufen, der seinen Dienst in Mannheim am 1.10.1975 antrat. Er schloss das Medizinstudium 1958 in Zürich ab, wo er 1960 mit einer experimentellen Arbeit aus der Gerinnungsphysiologie promovierte. Die Facharztausbildung erfuhr er an der Dermatologischen Universitätsklinik Zürich (Prof. Hans Storck) 1960-1965, um anschließend als Oberarzt an die Universitäts-Hautklinik Heidelberg (Prof. Urs W. Schnyder) zu wechseln. Im Jahre 1968 erfolgte die Habilitation an der Universität Heidelberg mit einem Thema aus der Lichtbiologie und 1973 die Ernennung zum apl. Professor. Mit seiner Berufung erhielt die Mannheimer Hautklinik erstmals einen Leiter, der keinen venerologischen Schwerpunkt vertrat. Damit wurde dem Rückgang der Venerologie Rechnung getragen und auch dem Prinzip der Komplementarität zwischen den beiden medizinischen Fakultäten Heidelberg und Mannheim an der Universität Heidelberg. Die Venerologie wird fortan tatkräftig und kompetent vom Heidelberger Kollegen, Prof. Detlef Petzoldt, vertreten, der auch wesentliche Verdienste erwarb in der Bekämpfung der weltweiten Seuche AIDS.
Prof. Ernst G. Jung brachte einen Schwerpunkt der Lichtbiologie und Photodermatologie nach Mannheim, der klinisch und experimentell ausgebaut wurde. Im Dachgeschoss ist die Lichtabteilung zur Diagnostik und Therapie mit verschiedensten Lichtquellen eingerichtet worden und auch die dazugehörigen experimentellen Labors. Die Bettenzahl wurde auf 68 reduziert und die Ambulanz ausgeweitet. Die Forschungsprogramme über erbliche und erworbene Lichtdermatosen, insbesondere über Xeroderma pigmentosum (seltene Genodermatose mit Lichtempfindlichkeit, multiplen Hauttumoren und einem molekularen Defekt der Exzisionsreparatur) wurden vom Sonderforschungsbereich Nr. 36 (Klinische Genetik) in denjenigen zur Krebsforschung ( $\mathrm{Nr}$. 136) übernommen und nach Mannheim überführt [18]. Photodermatologie, klinische Genetik und Dermato-Onkologie wurden verknüpft und zu einem überregionalen Referenzzentrum entwickelt, was sich in vielen wissenschaftlichen Tagungen niederschlug, zahlreiche internationale Gäste anzog und durch Stadt und Universität Ulm mit der Verleihung des Gottron-JustWissenschaftspreises 1991 anerkannt wurde. Eine Vielzahl wissenschaftlicher Verbindungen entstand im Rahmen des Wissenschaftsplatzes Rhein-Neckar, besonders mit den Kollegen am Deutschen Krebsforschungszentrum (DKFZ) in Heidelberg. Als Forum diente die Deutsche Gesellschaft für Lichtforschung (DGfL), welcher er 1985-1995 als deren Präsident diente.

Prof. Ernst G. Jung war Gründungsmitglied der Arbeitsgemeinschaft Dermatologischer Forschung (ADF) der DDG und auch der European Society for Dermatological Research (ESDR), welcher er als Vorstandsmitglied 1973-1977 und als Präsident 1976/77 diente. Mannheim war wesentlich an der Etablierung der experimentellen Dermatologie im Deutschen Sprachraum und in Europa beteiligt.

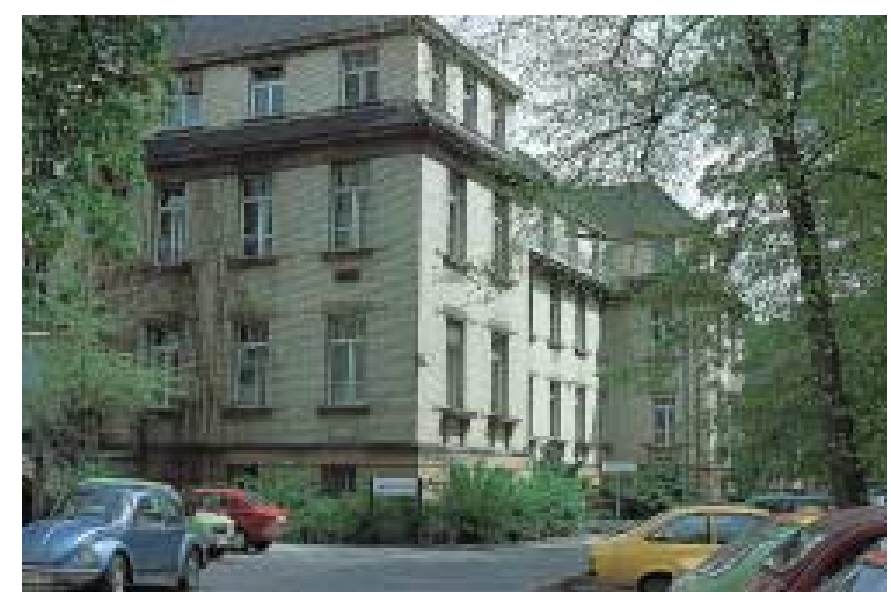

Abb. 5 Die Hautklinik Mannheim im Frühjahr 1977.

Im Jahre 1985 konnte der Pavillon der Hautklinik, dessen Pläne noch von 1913 stammen, von Grund auf saniert werden, wobei neben neuen Fenstern und der Einrichtung von Nasszellen, die großen Säle in Krankenzimmer mit 2-4 Betten aufgelöst wurden. In Übereinstimmung mit der landesweiten Krankenhausplanung wurde die Bettenzahl auf 50 festgesetzt (Abb. 5 u. 6). Gleichzeitig wurde die Ambulanz ausgeweitet und strukturiert sowie die Allergieteststelle räumlich verselbständigt, mit Forschungslabors ausgerüstet und als Kern der interdisziplinären Umweltmedizin etabliert. Photodermatologie, Allergologie und Hauttoxikologie 


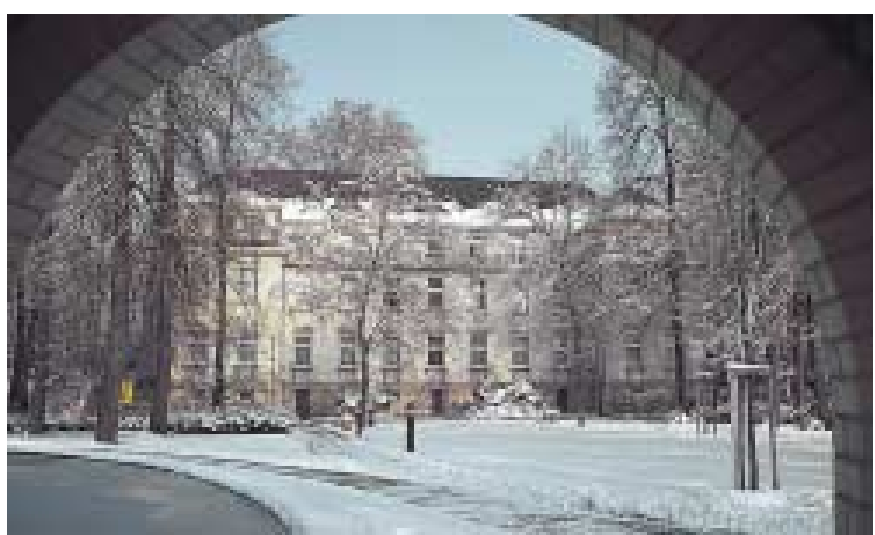

Abb. 6 Ansicht der Hautklinik Mannheim 1985, gesehen aus dem winterlichen Park.

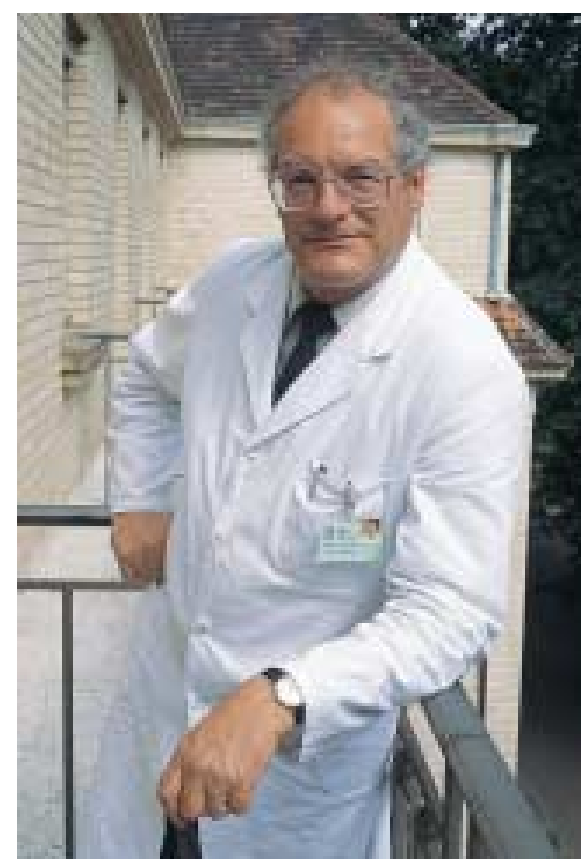
1992 auf dem Balkon
Abb. 7 Prof. Dr. med. Ernst G. Jung, im Dachgeschoss.$$
\text { im Dachgeschoss. }
$$

Prof. Ernst G. Jung hat 77 Doktoranden betreut und 6 junge Kollegen (darunter sind 3 Damen) bei der Habilitation begleitet, über 100 Assistenten ausgebildet und die leitenden Oberärzte erfolgreich zur Selbständigkeit geführt: Gerd Lischka wurde1979 als Professor für Dermatologie, Allergologie und klinische Immunologie an die Universität Tübingen berufen, Volker Voigtländer wurde 1990 zum Chefarzt der Hautklinik Ludwigshafen gewählt und Ingrid Moll folgte 1997 dem Ruf auf das Ordinariat unseres Faches an die Universität Hamburg (UKE). Mit seinen Mitarbeitern hat er die gesammelte Lehrerfahrung 1989 im studentischen Lehrbuch „Dermatologie“ der Dualen Reihe des Thieme Verlags, Stuttgart herausgegeben. Dieser „Marktführer“ [19] wird 2002 in der 5. und überarbeiteten Auflage erscheinen.

Zusammen mit dem DKFZ in Heidelberg (Prof. Harald zur Hausen) konnte er eine Dermato-Onkologische Kooperationseinheit neu schaffen, die experimentelle Ergebnisse umgehend und in enger Verknüpfung den Patienten in der Klinik zu Nutzen bringt. Diese wurde an der Hautklinik Mannheim angesiedelt, der HautAmbulanz benachbart und mit Forschungslabors reichhaltig ausgerüstet. Als Leiter konnte Herr Prof. Dr. med. Dirk Schadendorf aus Berlin berufen werden, der seinen Dienst in Mannheim am 1.4. 1997 antrat, zunächst den Aufbau der Einheit bewältigte, in der Melanomforschung große Erfolge verzeichnet und mit der Immuntherapie fortgeschrittener Melanome ganz neue, äußerst hoffnungsvolle Wege geht.

Damit sind die hauptsächlichsten ambulanten Bereiche, HautAmbulanz, Dermato-Onkologie und Allergieteststelle nahe beisammen im Erdgeschoss und im ersten OG des Bau 26 gruppiert, da wo vor 50 Jahren die Stationen der geschlechtskranken Prostituierten waren.

Der Wandel im Krankengut bringt auch einen eklatanten Wechsel der Ausrichtung und Nutzung der Räume, wobei hier nicht nur Flexibilität darzustellen ist, sondern richtungweisende Voraussicht.

Prof. Ernst G. Jung ist seit 1981 ordentliches Mitglied der Heidelberger Akademie der Wissenschaften und seit 1997 korrespondierendes Mitglied der Schweizerischen Akademie der medizinischen Wissenschaften. Seit 2 Jahrzehnten sitzt er in den Auswahlgremien zur Vergabe photobiologischer und photomedizinischer Preisees und er sitzt seit der Stiftung 1989 der Jury des Arnold Rikli Preis vor, der alle 2 Jahre für eine herausragende und richtungweisende wissenschaftliche Arbeit über die biopositiven Wirkungen von Licht vergeben wird. Die Preisvergabe erfolgt anlässlich der Symposien „Biologic Effects of Light“, die alternierend in Basel und USA im 2-jährigen Rhythmus abgehalten und von Michael F. Holick (Boston) und Ernst G. Jung (Mannheim) organisiert und durchgeführt werden.

Seit 5 Jahren ist das Klinikum Mannheim als gGmbH eigenverantwortlich, auch in finanziellen Angelegenheiten, und das Land Baden-Württemberg trägt, über die Universität Heidelberg, die Fakultät für klinische Medizin Mannheim als vertraglicher Partner. Überlegungen zu Kosten, Budget, Wirtschaftlichkeit und monatliches Controlling nehmen Einzug in den Alltag der Krankenversorgung und beherrschen, manchmal sogar übergebührlich, ärztliches Denken und Handeln. Auch die Mittel für klinische und experimentelle Forschungsvorhaben werden budge-
Er stellte sich der akademischen Selbstverwaltung zur Verfügung, langjährig als Mitglied des akademischen Senates der Universität Heidelberg, 1977-1980 als Dekan der Fakultät für klinische Medizin Mannheim und fünf Jahre als deren Studiendekan. Im Jahre 1981 hat er die Ethikkommission in Mannheim (jetzt die Ethikkommission 2 der Universität Heidelberg) gegründet und leitete diese während vieler Jahre. Zudem diente er der Universität Heidelberg von 1995-1997 als Prorektor für den Medizinbereich im Rektorat des Juristen Prof. Dr. Peter Ulmer. 
tiert und von der Fakultät leistungsabhängig vergeben. Diese Instrumente setzen Grenzen, geben aber auch eine gewisse Planungssicherheit. Das Drittmittelaufkommen für begutachtete Projekte und auch für Dienstleistungen wird ein wesentliches Kriterium zur Bewertung der Leistungsfähigkeit und damit der jährlichen Mittelzuteilung durch die Fakultät, stellt aber auch den „extrabudgetären“ Freiraum dar, den es zu nutzen gilt. Ist es verwunderlich, dass sogleich die Neider auf den Plan gerufen werden?

Die Hautklinik Mannheim hat diese neuen Herausforderungen als Chance gesehen und zusätzlich zu den Forschungsprojekten ein klinisches Prüfzentrum für Dermatika aller Couleur, für Kosmetika und auch für Körperpflegemittel etabliert. Sie ist damit recht erfolgreich geworden und hat sich in den vergangenen drei Jahren als die Klinik mit dem größten Drittmittelaufkommen der Fakultät ausgewiesen. Damit ist es gelungen, die Zahl der wissenschaftlichen Mitarbeiter an der Hautklinik Mannheim beinahe zu verdoppeln. Dadurch kann nicht nur die Vertiefung, sondern auch die Breite und die Vielfalt wissenschaftlicher Tätigkeiten erreicht werden. Prof. Ernst G. Jung hat sich am 11. 2. 2000 von seinen Studenten, Kollegen, Freunden und Patienten mit der Abschiedsvorlesung „Resonanz und Raison“ bedankt und verabschiedet und er wurde am 31.3. 2000 emeritiert. Er lebt weiterhin in Heidelberg und nimmt am akademischen Leben teil.

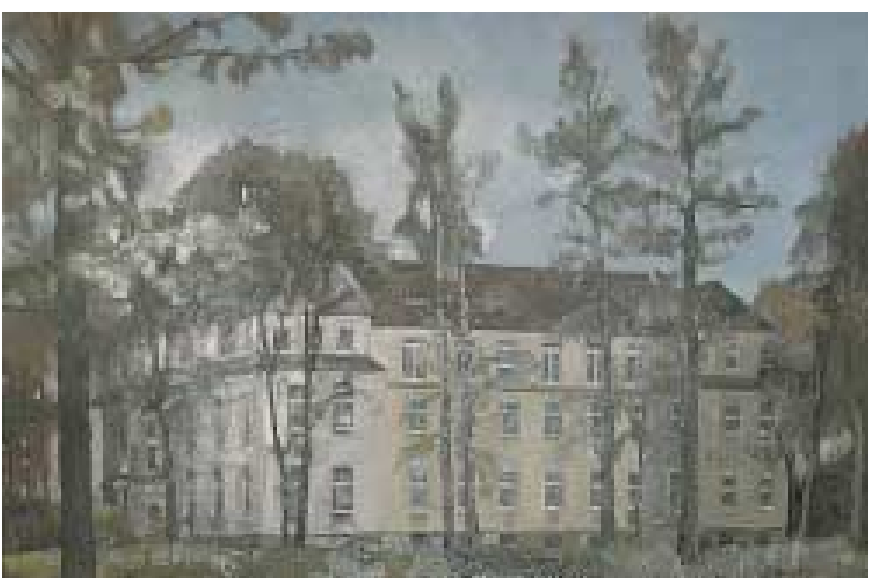

Abb. 8 Die Hautklinik des Klinikums Mannheim vom Park aus, 1993. Öl auf Kunststoff, gemalt von Wolf Magin, Mannheim (geb. 1927).

Als Nachfolger konnte Herr Prof. Dr. med. Sergij Goerdt (geb. am 14. 5.1959 in Münster) aus Berlin auf den ordentlichen Lehrstuhl für unser Fach berufen werden, der die Klinik übernahm und, den modernen Vorstellungen entsprechend, in „Universitätsklinik für Dermatologie, Venerologie und Allergologie“ umbenennen ließ. Er hat es auch durchgesetzt, dass die Dermatologie in Mannheim nach gut 100 Jahren, welche sie jetzt 80 Jahre in demselben Pavillon (Abb.8), wenn auch mit wiederholten „Verjüngungskuren“, verbrachte, die Chance eines Neubaus erhält, der für die künftigen Bedürfnisse unseres Faches konzipiert wird. Der Neubau der Dermatologie ist im Park des Klinikums, zwischen Kinderzentrum und Hautklinik-Altbau, geplant mit Baubeginn schon im Jahre 2003. Dazu gratulieren alle und wünschen viel Glück.

\section{Überblick}

Die 100 Jahre Dermatologie in Mannheim sind gekennzeichnet durch Konstanz, Kompetenz, Flexibilität und Innovation. Die Konstanz wird repräsentiert durch die 4 Chefärzte, welche großen Zeitabschnitten eine persönliche Prägung gaben und zuverlässige Fachkompetenz zur Verfügung stellten. Dies lässt sich anhand der Venerologie besonders gut darstellen und der engagierten und innovativen Art der Therapie von Syphilis und Gonorrhö. Dies erfolgte unter frühzeitiger Nutzung der jeweils neuen Therapieprinzipien, Salvarsan im ersten Jahrzehnt und Penicillin im fünften. Die Hautklinik Mannheim konnte nicht nur die venerologischen Bedürfnisse der Patienten aus ihrer Stadt bestens abdecken, sondern auch den enormen Andrang an zugereisten Patienten in den zwei Nachkriegsperioden bewältigen. Dazu bedurfte es nicht nur großen Könnens und letzten Einsatzes der Ärzte, sondern auch innovativer Gestaltungskraft der Dermatologie und des ganzen Krankenhauses. Mannheim war wesentlich beteiligt an der Einführung und Optimierung der venerologischen Behandlungen mit modernen Antibiotika und hat damit kräftig zur Bewältigung der Epidemien und zum Rückgang venerologischer Erkrankungen beigetragen. Die langen stationären Behandlungen wurden überflüssig, die Betten konnten schrittweise reduziert werden. Weite Bereiche der Venerologie werden nun ambulant bedient und fallen in die Kompetenz der niedergelassenen Hautfachärzte. Diese sind, an der Klinik gut ausgebildet, dazu auch bestens in der Lage. Die Versorgung der Bevölkerung der Stadt Mannheim mit Hautfachärzten hat sich, mit einem kurzen Defizit im ausgehenden Kaiserreich, eingependelt auf einen Hautarzt zur Versorgung von 20000 Einwohnern (Tab. 1).

Die zweite Hälfte der 100 Jahre Dermatologie in Mannheim ist gekennzeichnet durch diese Wandlung und innovative Zuwendung zu Zukunfts-Feldern, die in der Dermatologie der Berufswelt und in derjenigen des Freizeitbereiches liegen. Der Begriff „Umweltdermatologie“ fasst alles zusammen: Allergologie, Toxikologie, Berufsdermatologie, Photobiologie, Lichtdermatologie etc. und die damit verbundene Dermato-Onkologie, die besonders an Bedeutung gewinnt. Diese erworbenen Hauterkrankungen und Gefährdungen verlangen auch eine Prävention und Früherkennung, und sie sind komplementär zu sehen zu den vielfältigen erblichen Hautkrankheiten oder erblichen Dispositionskrankheiten der Haut und der Schleimhäute, die häufig (Atopie, Psoriasis, Ichthyosen etc.) oder eher selten sind und sowohl Herausforderung als auch Modell darstellen. Dazu kommt

Tab. 1 Niedergelassene Hautfachärzte in der Stadt Mannheim [5]

\begin{tabular}{|cccc|}
\hline Jahr & Hautärzte & Einwohner in $\mathbf{M A}$ & $\mathbf{1}$ Hautarzt pro Einw. \\
\hline 1871 & - & 40000 & - \\
\hline 1892 & 1 & & \\
\hline 1901 & 7 & 168000 & 24000 \\
\hline 1914 & 6 & 220000 & 36000 \\
\hline 1933 & 13 & 275000 & 21000 \\
\hline 1949 & 11 & 240000 & 22000 \\
\hline 1975 & 14 & 322000 & 23000 \\
\hline 2000 & 16 & 320000 & 20000 \\
\hline
\end{tabular}

Nicht berücksichtigt sind die Fach- und Chefärzte der Hautklinik und des staatlichen Gesundheitsamtes Mannheim. 
die Vielzahl allergologischer und immunologischer Krankheiten, die sich wesentlich am Hautorgan abspielen. Neben dem Stock von 50 Betten, die erfahrungsgemäß dem Bedarf genügen dürften, war eine beträchtliche Ausweitung der ambulanten Bereiche und Dienste in der Hautklinik notwendig, insbesondere um neue und noch risikobelastete Verfahren anzubieten, neue Entwicklungen zu etablieren, besondere Bereiche zu bedienen, sowie den niedergelassenen Kollegen als Referenz, den Patienten als zweite Meinung, zur Verfügung zu stehen. Eine enge Verzahnung mit den niedergelassenen Fachkollegen und auch mit den anderen Fächern im Klinikum ist notwendig und bedarf der besonderen Pflege in der Patientenbetreuung, in der Fortbildung, mit Fallbesprechungen und durch viele persönliche Kontakte. So ist die Dermatologie in Mannheim nach 100 Jahren Konstanz, Kompetenz, Innovation und weitblickendem Wandel ausgesprochen reif und bereit, in einem eigens dafür konzipierten und ab 2003 zu errichtenden Neubau im Park des Klinikums adäquat untergebracht zu werden. Damit werden die Grundlagen geschaffen für eine fruchtbare Weiterentwicklung, sowohl zur optimalen Versorgung der Mannheimer Bevölkerung, als auch zur Bewältigung der universitären Aufgaben in Lehre und Forschung, Voraussetzung zum hohen Standard ärztlicher Kunst als Zukunftsinvestition.
Literatur

${ }^{1}$ Volz R. Das Spitalwesen und die Spitäler des Großherzogtums Baden. Karlsruhe: Malsch und Vogel, 1861

${ }^{2}$ Schmidt W. Zur Entwicklung der Städtischen Krankenanstalten Mannheim vom Nothaus bis zur Errichtung fachärztlich geleiteter Abteilungen. Med Mannheim 1978; 1: $33-40$

${ }^{3}$ Schmidt W. Über Ärzte und medizinische Einrichtungen in Mannheim zur Zeit des Kurfürsten Karl-Theodor. Med Mannheim 1977; 3: 27-34

${ }^{4}$ Schmidt W. Chefärzte der Städt. Krankenanstalten Mannheim in den Jahren 1884-1964 und ihre Beziehungen zu Medizinischen Fakultäten. Heidelberg: Ruperto Carola, 1980; 32: 43-51

${ }^{5}$ Gawliczek OH, Senk WE, Hatzig H. Bezirksärztekammer Nordbaden, Ärzteschaft Mannheim (Hrsg). Chronik der Ärzte Mannheims. 350 Jahre Medizin in der Stadt der Quadrate. 1978

${ }^{6}$ Friedmann M. In memoriam Heinrich Loeb. Ärztl. Mitteilungen aus und für Baden. 1932; 86: 138

${ }^{7}$ Lion V. In memoriam Heinrich Loeb. Zbl H \& G 1932; 41: 287- 288

${ }^{8}$ Hermann F. Friedrich Schmidt-LaBaume zum 65 . Geburtstag. Hautarzt 1957; 8: 191 - 193

${ }^{9}$ Schmidt W. In memoriam Friedrich Schmidt-LaBaume 1892-1973. Hautarzt 1974; 25: 411

${ }^{10}$ Schmidt-LaBaume F, Lietz G. Die Emulsionen in der Hauttherapie (2. Aufl. 1951). Stuttgart: Hirzel, 1943

${ }^{11}$ Czetsch-Lindenwald H, Schmidt-LaBaume F. Salben - Puder - Externa, die äußeren Heilmittel der Medizin (3. Aufl. 1950; Ergänzung 1956). Berlin, Heidelberg: Springer, 1939

${ }^{12}$ Hoffmeister W. Das Klinikum Mannheim der Universität Heidelberg. Welt am Oberrhein (1970; 3:135-142). Heidelberg: Ruperto Carola, 1971; 49: $210-220$

${ }^{13}$ Bleyl U, Jung EG. 25 Jahre Mannheimer Medizinische Fakultät der Universität Heidelberg. Mannheimer Hefte, 1989; 2: 78-87

${ }^{14}$ Lischka G. Zur Emeritierung Werner Schmidts. Z Hautkrh 1976; 51: $1-2$

${ }^{15}$ Lischka G, Jung EG. Emeritierungsfeier für Prof. Dr. Werner Schmidt in Mannheim. Hautarzt 1976; 27: 506

${ }^{16}$ Gartmann H. Werner Schmidt zum 65. Geburtstag. Hautarzt 1972; 23 : $190-191$

17 Jung EG. In memoriam Werner Schmidt 1907-1989. Hautarzt 1989; 40: 789

${ }^{18}$ Brossmer R, Jung EG, Werner D. Der Sonderforschungsbereich „Krebsforschung“ an der Universität Heidelberg. Heidelberg: Ruperto Carola, 1986: 74

${ }^{19}$ Jung EG. Dermatologie, Lehrbuch der Dualen Reihe. 4. Auflage. Stuttgart: Thieme, 1998 